\title{
Miasis entérica por Hermetia illucens (Diptera: Stratiomyidae) en una paciente geriátrica de Costa Rica
}

\author{
OLGER CALDERÓN-ARGUEDAS*, JOSÉ MURILLO BARRANTES** y MAYRA E. SOLANO*
}

\section{ENTERIC MYIASIS BY Hermetia illucens (DIPTERA: STRATIOMYIDAE) IN A GERIATRIC PATIENT OF COSTA RICA}

We report a case of enteric myiasis by Hermetia illucens (Diptera: Stratiomyidae) in a 71 year old woman. The patient showed history of ischemic hearth disease and hypertension. She also suffered colelitiasis and diverticulitis with high eosinophilia and recently there was diagnosed an abdominal carcinomatosis process. The eosinophil levels were between 9 to $31 \%$ in different analysis. Considering the presence of Ascaris lumbricoides eggs in a fecal sample, she was treated with mebendazole. After the treatment, two larvae compatible with $\boldsymbol{H}$. illucens (Diptera: Stratiomyidae) were defecated. This is the first report of an enteric myiasis by $\boldsymbol{H}$. illucens in Costa Rica.

Key words: Hermetia illucens. Stratiomyida. Enteric myiasis. Diptera. Costa Rica.

\section{INTRODUCCIÓN}

Las miasis consisten en infestaciones de órganos y tejidos de animales vertebrados ocasionadas por larvas de muscoideos que al menos por un período de tiempo se ubican y alimentan de tejidos vivos o necróticos ${ }^{1}$.

De acuerdo a una clasificación ${ }^{2}$, las miasis pueden ser agrupadas de acuerdo a la parte del cuerpo que se ve afectada. De esta forma, se reconocen las traumáticas, las cuales se relacionan con heridas abiertas, las de la nariz, boca y senos accesorios, las de la región anal y la vagina, las de la vejiga y conductos genitourinarios, las furunculares (dérmicas y subdérmicas) y las entéricas (gastrointestinales, gástricas e intestinales) $)^{2}$.

Hermetia illucens es una especie nativa, ampliamente distribuida en la América Tropical ${ }^{3}$. Sus larvas pueden ocupar muchos tipos de substrato como materia vegetal en descomposición, excremento y en algunos casos se han informado como parte de la necrofauna prevalente en

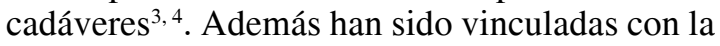
ocurrencia de miasis dérmica y entéricas ${ }^{3,5}$.

El presente caso clínico es el primer informe en la literatura biomédica costarricense que describe un caso de miasis entérica humana por H. illucens.

\section{CASO CLÍNICO}

Paciente femenina, 71 años, vecina de Cañas (Provincia de Guanacaste). Desde 1999 empezó a recibir control médico en el Hospital de Upala localizado en la Zona Norte del País, ya que

\footnotetext{
* Centro de Investigación en Enfermedades Tropicales (CIET), Departamento de Parasitología, Facultad de Microbiología, Universidad de Costa Rica. E-mail: olgerc@ cariari.ucr.ac.cr

** Servicio de Cirugía, Hospital Dr. Enrique Baltodano, Caja Costarricense de Seguro Social.
} 
presentó un cuadro de cardiopatía isquémica e hipertensión de alto riesgo. En los exámenes control llamó la atención la ocurrencia de una marcada eosinofilia con valores que iban desde el 9 al 31\% del diferencial de glóbulos blancos. En el año 2004 se le diagnosticó colelitiasis y colecistitis, los cuales se vincularon en ese momento con dolor abdominal intenso localizado en el hipocondrio derecho. En el año 2005 fue diagnosticado un cuadro de diverticulitis con dolor en hemiabdomen inferior recurrente. La paciente persistió con su marcada eosinofilia (22\%), mostrándose el abdomen doloroso y mostrando un cuadro de estreñimiento severo. A pesar de la necesidad de intervenir quirúrgicamente para evitar complicaciones ulteriores, la paciente se negó en este momento a que se le practicara cualquier procedimiento. Durante uno de los internamientos posteriores el examen de frotis fecal mostró la presencia de huevos de Ascaris lumbricoides por lo que se le trató con Mebendazole. Posterior a la ingesta del fármaco, la paciente defecó dos larvas muy grandes. El material fue fijado en alcohol al $70 \%$ y aclarado en lactofenol por siete días. Posteriormente se montó entre lámina y laminilla, utilizando Medio Hoyer como medio de montaje.

Las larvas presentaron una longitud de aproximadamente $45 \mathrm{~mm}$ y mostraron una morfología vermiforme hemicéfala (Figura 1). Las características observadas son compatibles con las de las larvas de $H$. illucens ${ }^{2}$.

Posteriormente, la paciente fue intervenida quirúrgicamente, proceso en el que se evidenció un cuadro de carcinomatosis generalizada a través de todo el peritoneo con una perforación punctiforme del ciego. Una gran masa que involucraba la vesícula, el ángulo hepático del colon y el epiplón mayor se encontraba generando obstrucción abdominal que ameritó una ileostomía descompresiva de la zona.

Mediante una búsqueda bibliográfica basada en el Índice de Publicaciones Entomológicas de Costa Rica ${ }^{6}$ y PubMed ${ }^{7}$ se pudo constatar que no existen registros de casos de miasis entéricos por H. illucens en la literatura médica de Costa Rica.

\section{DISCUSIÓN}

La observación de larvas de muscoideos en materias fecales usualmente se asocia con las

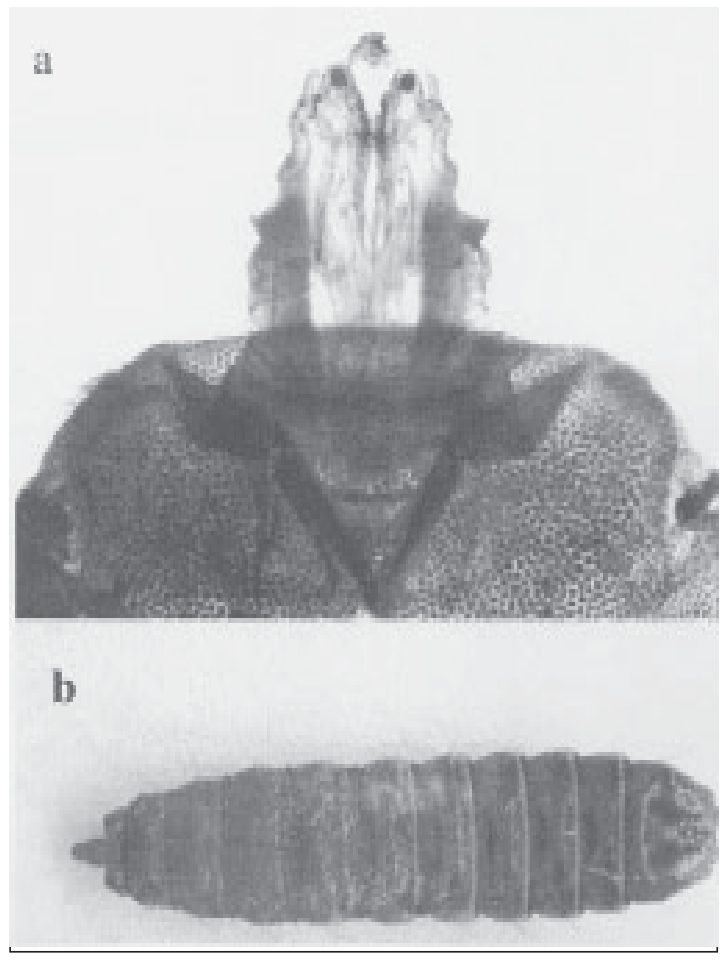

Figura 1. Larva de cuarto estadio de Hermetia illucens procedente de la paciente referida. a: región anterior (barra $=1,3 \mathrm{~mm}) ; \mathbf{b}$ : vista macroscópica.

pseudomiasis ${ }^{1,2}$. A diferencia de las miasis entéricas verdaderas como las provocadas por moscas del género Gasterophilus que ocurren en equidos $^{1,2}$, el término pseudomiasis se aplica al cuadro clínico que se deriva de la ingesta accidental por tracto gastrointestinal de larvas de mosca, el cual puede o no acompañarse de sintomatología ${ }^{2}$. Las larvas suelen encontrarse viables luego de su eliminación en materias fecales, lo que refleja su capacidad de resistencia y no necesariamente su potencial adaptativo ${ }^{2}$. En el caso que se presenta las larvas eliminadas correspondieron a formas de cuarto estadio de H. illucens. Estas larvas presentaron un tamaño bastante grande (Figura 1), lo que refleja la imposibilidad de que éstas hayan sido ingeridas en este estadio de desarrollo. Este hecho podría sugerir la posibilidad de que la miasis hubiese iniciado con la ingestión de huevos o larvas de los primeros estadios, los cuales experimentaron 
un progreso en su desarrollo internamente en el hospedador. En este sentido, diversos autores han reconocido a $H$. illucens como una agente causal de miasis entéricas verdaderas en animales y humanos ${ }^{1-5}$. Es así como autores informaron acerca de un caso de miasis entérica por $H$. illucens en un bovino macho de la raza Simmental en el Municipio de Sucilá, Yucatán, México, el cual presentaba un cuadro gastroentérico con timpanismo crónico que progresó hasta provocarle la muerte ${ }^{8}$. Luego de la necropsia se encontraron en el rumen 20 larvas del díptero aparentemente adheridas a la mucosa intestinal. La mucosa se encontró con un aspecto eritematoso petequial ${ }^{8}$. Otros autores describieron una miasis entérica por el mismo agente en una niña de 7 años de edad, la cual expulsó por medio del vómito 2 larvas de la mosca ${ }^{5}$.

Dado que las larvas pueden ocupar múltiples ambientes para su desarrollo de los cuales se pueden citar frutas sobre maduras, materia vegetal en descomposición, excremento, cadáveres expuestos y panales de abejas abandonados ${ }^{3}$, las posibilidades de ingestión de formas larvales o huevos son muy variadas. En el presente caso, la ocurrencia de eosinofilias altas y el hallazgo de huevos de A. lumbricoides en un frotis fecal, refleja la posibilidad de que el ambiente donde la paciente se maneja así como sus hábitos higiénicos resulten permisivos para las parasitosis gastrointestinales y por consecuencia la asociación de $H$. illucens con materia fecal podrían explicar la ocurrencia del cuadro de miasis presentado.

Clínicamente el problema de diverticulitis posiblemente favoreció el establecimiento de las formas larvales de $H$. illucens, ya que los divertículos podrían figurar como sitios de adhesión del díptero a la mucosa intestinal. La situación de carcinogénesis que entre otras cosas contribuyó a la formación de una masa que produjo obstrucción intestinal constituye uno de los factores que podrían haber explicado el estreñimiento que sufrió la paciente. En estas condiciones de tránsito intestinal lento, las posibilidades de permanencia de organismos de vida libre en el lumen intestinal se incrementa.

Se requiere conocer más acerca de la biología de $H$. illucens y de los cuadros que puede desencadenar a fin de poder prevenir la ocurrencia de miasis como la descrita en el presente caso clínico.

\section{RESUMEN}

Se reporta un caso de miasis entérica por Hermetia illucens (Diptera: Stratiomyidae) en una paciente femenina de 71 años de edad. La paciente presentó antecedentes de cardiopatía isquémica e hipertensión. También sufrió de colelitiasis y diverticulitis con una alta eosinofilia y recientemente se le diagnosticó un proceso carcinomatoso abdominal. Los niveles de eosinófilos estuvieron entre el 9 y el $31 \%$ en diferentes análisis. Considerando la presencia de huevos de Ascaris lumbricoides en una muestra fecal, fue tratada con mebendazole; luego del tratamiento la paciente defecó dos larvas, cuya morfología es compatible con H. illucens. El presente trabajo constituye el primer informe de una miasis entérica por H. illucens en Costa Rica.

\section{REFERENCIAS}

1.- HARDWOOD R, JAMES M. Myiasis. En: Entomology in human and animal health. $7^{\text {th }}$ edition. The Macmillan Publishing Co. Inc. New Yor, 1979. 548 pp.

2.- JAMES M T. The flies that cause myiasis in man. US Dept Agric Misc Publ 1947. 175 pp.

3.- JIRÓN L F, SOLANO M E. Notes on the eclectical feeding preferences of the black soldier fly Hermetia illucens (Diptera: Stratiomyidae) in Costa Rica. Brenesia 1988; 30: 101-3.

4.- TURCHETO M, LAFISCA S, COSTANTINI G. Postmortem interval (PMI) determined by study sarcophagous biocenoses: three cases from the provence of Vence (Italy). For Sci Int 2001; 120: 28-31.

5.- LEE H L, CHANDRAWATHANI P, WONG W Y, et al. A case of human enteric myiasis due to larvae of Hermetia illucens (Family: Stratiomyiadae): first report in Malaysia. Malays J Pathol 1995, 17: 109-11.

6.- JIRON L F, SANCHO DE BARQUERO M. Índice de publicaciones entomológicas de Costa Rica. Consejo Nacional de Investigaciones científicas y tecnológicas (CONICIT)/Organization of Tropical Studies (OTS)/ Universidad de Costa Rica (UCR). Primera Edición, San José, Costa Rica. 1983. 305 pp.

7.- http://www.ncbi.nlm.nih.gov/entrez/query.fcgi.

8.- MANRIQUE-SAIDE P, RODRÍGUEZ-VIVAS R, QUIÑONES-RODRÍGUEZ M, QUIROZ-APARICIO R. Un caso de pseudomiasis por larvas de Hermetia illucens (Diptera: Stratiomyidae) en un bovino. Rev Biomed 1999; 10: 173-6.

Agradecimientos: Los autores desean externar su agradecimiento a la Dra. Ligia Carballo del Hospital de Upala (CCSS) por la colecta del material entomológico y al Dr. Miguel Ángel Cabrera, director del Laboratorio Clínico del mismo Hospital, por su colaboración en la documentación del caso. También se agradece a la Sección de Extensión Docente de la Vicerrectoría de Acción Social de la Facultad de Microbiología por su apoyo logístico al proyecto ED 548. 